\title{
Association of Hypothyroidism with Infrequent Tension-Type Headache
}

\author{
Rafiqul Islam¹, Monsur Habib², Farida Khatun³, Uzzwal Kumar Mallick, Md. Mamnur Rashid5, \\ Mahmudul Islam ${ }^{6}$, Shapon Kumar Ghosh ${ }^{7}$, Kamal Ahmed ${ }^{8}$ \\ ${ }^{1}$ Associate Professor, Department of Pharmacology, National Institute of Neurosciences \& Hospital, Dhaka, Bangladesh; ${ }^{2}$ Former \\ Head \& Professor of Neurology, Dhaka Medical College, Dhaka, Bangladesh; ${ }^{3}$ Associate Professor, Department of Physical \\ Medicine \& Neurorehabilitation, National Institute of Neurosciences \& Hospital, Dhaka, Bangladesh; ${ }^{4}$ Assistant Professor, \\ Department of Critical Care Medicine, National Institute of Neurosciences \& Hospital, Dhaka, Bangladesh; ${ }^{5}$ Assistant \\ Professor \& Resident Physician, Department of Neurology, National Institute of Neurosciences \& Hospital, Dhaka, \\ Bangladesh; ${ }^{6}$ Assistant Professor, Department of Neurology, National Institute of Neurosciences \& Hospital, Dhaka, \\ Bangladesh; ${ }^{7}$ Associate Professor, Department of Neurology, Dhaka Medical College, Dhaka, Bangladesh; \\ ${ }^{8}$ Professor, Department of Neurology, North East Medical College, Sylhet, Bangladesh
}

[Received on: 22 November 2020; Accepted on: 12 December 2020; Published: 1 January 2021]

\begin{abstract}
Background: Tension-type headache is a common neurological problem among general population of Bangladesh. Objective: This study was undertaken to ascertain the frequency of subclinical and over hypothyroidism in patients with infrequent TTH. Methodology: This study was conducted in a tertiary care hospital Dhaka medical college in Bangladesh. The study subjects consisted of 200 patients primary headache disorders. Thyroid function test were performed. All four sub-types of tension type headache were considered to include in the study, namely: Infrequent episodic (or infrequent), frequent episodic (or frequent), chronic sub-type and probable sub-type. Result: Forty percent (40\%) patients in this study had infrequent TTH, 30\% had frequent episodic TTH, 21\% had chronic sub-type of TTH and 9\% patients had probable sub-type of TTH. overt hypothyroidism. Among 46 patients with frequent episodic TTH 10 patients had subclinical hypothyroidism and 4 patients had overt hypothyroidism. Out of 18 patients with probable TTH 3 patients had subclinical hypothyroidism but no patient was overt hypothyroid. Out of 42 chronic TTH patients 12 had subclinical and another 12 had overt hypothyroidism. Statistically significant difference was seen among different sub-types of headache in relation to thyroid status. Conclusion: Hypothyroidism is found to be a co morbidity or precipitating factors to be development of infrequent type of tension-type headache. [Journal of National Institute of Neurosciences Bangladesh, January 2021;7(1): 60-64]
\end{abstract}

Keywords: : Hypothyroidism; Tension-Type Headache; TTH; neurological disorder

Correspondence: Dr. Rafiqul Islam, Associate Professor, Department of Pharmacology, National Institute of Neurosciences \& Hospital, Dhaka, Bangladesh; Cell no.:+8801924146535; Email: drrafiqulnins123@gmail.com

Conflict of interest: None

Funding agency: Any of the author did not receive any external funding for this study.

Contribution to authors: Islam $\mathrm{R}$ was involved in the concept, study design and data collection. The rest of the authors were involved in data collection and partially writing and editing the manuscript.

How to cite this article: Islam R, Habib M, Khatun F, Mallick UK, Rashid MM, Islam M, Ghosh SK, Ahmed K. Association of Hypothyroidism with Infrequent Tension-Type Headache. J Natl Inst Neurosci Bangladesh, 2021;7(1): 60-64

Copyright: C2021. Islam et al. Published by Journal of National Institute of Neurosciences Bangladesh. This article is published under the Creative Commons CC BY-NC License (https://creativecommons.org/licenses/by-nc/4.0/). This license permits use, distribution and reproduction in any medium, provided the original work is properly cited, and is not used for commercial purposes.

\section{Introduction}

Tension-type headache is a neurological disorder characterized by recurrent episodes of headache lasting 30 minutes to 7 days $^{1}$. The pain is typically pressing or tightening in quality, of mild to moderate intensity, and bilateral in location, and does not worsen with the routine physical activity. Nausea and vomiting is usually absent, but photophobia or phonophobia may be present ${ }^{2}$. These headaches were previously known by many terms such as psychogenic headache, stress headache, psychomyogenic headache, muscle contraction headache etc. However, the term "tension type headache" (TTH) 
has been chosen by the International Classification Headache Diagnosis I (ICHD I) and have been retained by ICHD $\mathrm{II}^{3}$.

Tension-type headache (TTH) is the most prevalent form of headache in all age groups across the globe $\mathrm{e}^{4}$ TTH leads to considerable disability with up to $60.0 \%$ of individuals reporting decreased work effectiveness, increased absenteeism and reduced social engagement ${ }^{5}$. The underlying cause of tension-type headache is uncertain. Activation of Hyper excitable peripheral afferent neurons from head and neck muscles might be the likely explanation for tension-type headache ${ }^{6}$. Muscle tenderness and psychological tension may aggravate tension-type headache but are not clearly its cause. Abnormalities in central pain processing and generalized increased pain sensitivity are present in some patients with tension-type headache?

There are several consistent studies have shown that patients with TTH had significantly higher proportion of subclinical hypothyroidism and overt hypothyroidism as compared to the control subjects ${ }^{8}$. It has been observed that improvement in headache occurs in $30 \%$ of hypothyroid patients after initiation of thyroid hormone replacement?. There have been few recent advances in treatment options for TTH. Non pharmacologic approach like avoidance of associated factors and reassurance has been proved useful in several studies ${ }^{10}$. As far the knowledge goes this will be the first study in Bangladesh to find out the relationship between hypothyroidism and tension -type headache. The overall response to treatment of TTH is often not satisfactory. For a majority of individuals with $\mathrm{TTH}$, the mainstay in pharmacotherapy is simple analgesics and non-steroidal anti-inflammatory drugs ${ }^{4}$. This study was undertaken to ascertain the frequency of subclinical and over hypothyroidism in patients with infrequent TTH.

\section{Methodology}

It was a cross sectional observational study. Data were collected from the department of Neurology (Headache Clinic), Dhaka Medical College Hospital, Dhaka, Bangladesh. Patient with tension-type headache attending in headache clinic in $\mathrm{DMCH}$ with the age group 18 to 55 years of age of both male and female with the diagnosis of tension type headache on the basis of ICHD-2 criteria were selected as study population. Patients with any form of thyroid disease prior to the enrolment in the study as revealed by clinical examination or past medical records or patients with abnormal neurological examination, pregnancy, any chronic illness known to affect thyroid hormone levels,
Chronic kidney disease or other systemic illness or chronic drug intake known to affect thyroid status of the patient like lithium carbonate, amiodarone and anti-thyroid drugs were excluded from this study. Non-probability purposive consecutive sampling method was used to select sample population. Data was collected from the respondents through interview. At first study subjects were screened according to inclusion and exclusion criteria who complaint headache. Then physical examination was done accordingly. Diagnosis of the TTH was made on the basis of ICHD-2 and verified by a consultant neurologist. Following confirmation demographic profile was collected by face-to-face interview by using a semi-structured questionnaire. In all cases informed written consent was taken after explaining the aims, objectives of the study in each patient. Researcher filled up whole of the questionnaire and patients were requested to perform thyroid function test from Institute of Nuclear Medicine and Allied Science (INMAS). All the study subjects were tested for serum free T4 and TSH by radio immune assay (RIA) and immune radio metric assay (IRMA) using radioisotope 1-125 as tracer. Personal contact numbers were collected during interview and patients were followed up in prefixed scheduled date. During follow up visit, hormonal level were included in their personal data sheet and researcher himself ascertained subtypes of hypothyroidism. Moreover, following primary screening endocrine abnormality were also confirmed by an endocrinologist. Subjects with incomplete data were excluded before final analysis. Also in case of hyperthyroidism the patients were excluded from the study and inclusion of another patient was done until it matched 200 samples. Based on TTH and serum T4 and TSH level, study population were divided into two groups designated as group I representing the patients with euthyroid and group II consisted with the patients with hypothyroidism either overt or subclinical. After collecting the data, it was checked and rechecked for omission, inconsistencies and improbabilities. After cleaning the data it was edited, coded and entered into the computer. Statistical analysis of the study was done by computer software device as the Statistical Package for Social Science (SPSS) version 22.0. Confidence interval was considered at $95 \%$ level. The qualitative variables were expressed as frequency and percentage and the quantitative variables were expressed as mean with standard deviation. During analysis chi-square test was done to estimate the relationship or association between TTH and hypothyroidism. P value less than 
0.05 was considered statistically significant.

\section{Results}

This study was conducted on 200 patients who met the ICHD-2 diagnostic criteria of Tension Type Headache. Mean age of the patients studied was 35.4 years $( \pm 9.9)$. Among the respondents, minimum age was 18 years and maximum age was 55 years. Majority of the patients $(31.0 \%)$ were in between 36 to 45 years (Table $1)$.

Table 1: Age Group of the Respondents $(n=200)$

\begin{tabular}{lcc}
\hline Age Group & Frequency & Percent \\
\hline 18 to 25 Years & 48 & 24.0 \\
26 to 35 Years & 50 & 25.0 \\
36 to 45 Years & 62 & 31.0 \\
46 to 55 Years & 40 & 20.0 \\
Total & $\mathbf{2 0 0}$ & $\mathbf{1 0 0 . 0}$ \\
\hline
\end{tabular}

Among 200 patients majority ( 80 patients, 40\%) had infrequent episodic tension type headache (TTH). Next to infrequent episodic TTH was frequent episodic TTH with number of $60(30 \%) .42(21 \%)$ patients had chronic sub-type of TTH and remaining 18(9.0\%) patients had probable sub-type of TTH (Figure I).

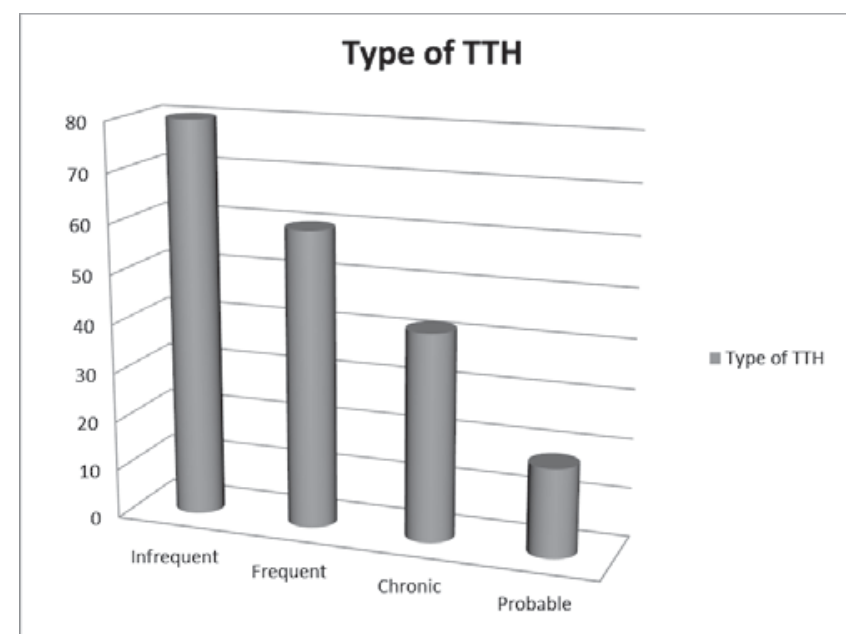

Figure I: Distribution of patients among sub-types of TTH $(\mathrm{n}=200)$
This study focused on patients of TTH who had hypothyroidism or normal thyroid status. As a result, patients who were found hyperthyroid were excluded from the study. Out of 200 patients with TTH total 61 patients $(30.5 \%)$ had hypothyroidism and rest of them (139 patients, $69.5 \%$ ) had normal thyroid status. Among the 61 hypothyroid patients subclinical hypothyroidism was found in 41 patients and 20 patients had overt hypothyroidism (Figure II).

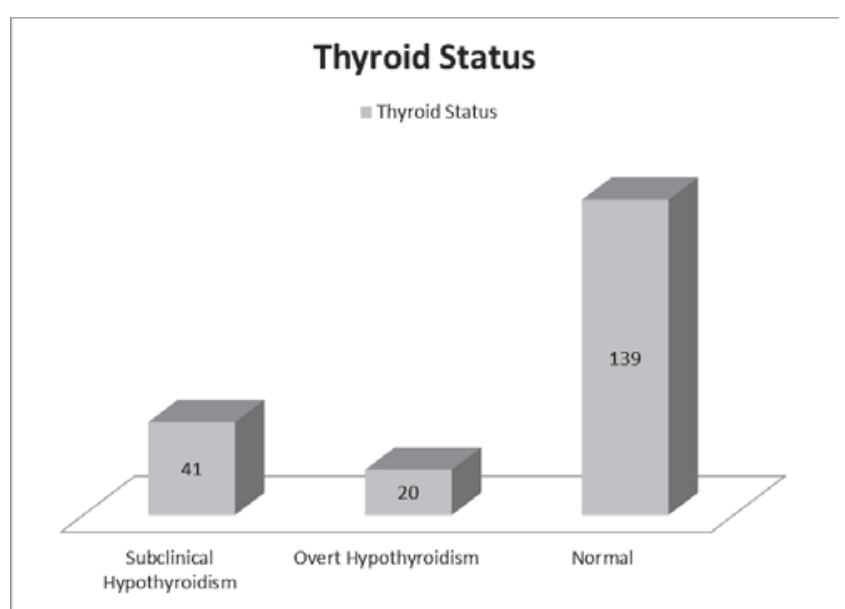

Figure II: Status of thyroid in TTH patients

Among 80 patients with infrequent episodic TTH 16 patients had subclinical hypothyroidism and 4 patients had overt hypothyroidism. Among 46 patients with frequent episodic TTH 10 patients had subclinical hypothyroidism and 4 patients had overt hypothyroidism. Out of 18 patients with probable TTH 3 patients had subclinical hypothyroidism but no patient was overt hypothyroid. Out of 42 chronic TTH patients 12 had subclinical and another 12 had overt hypothyroidism. Statistically significant difference was seen among different sub-types of headache in relation to thyroid status (Table 2).

\section{Discussion}

Patients with daily or frequent headache are commonly seen in neurology clinics. Most of them are diagnosed

Table 2: Thyroid status in relation of different sub-types of TTH $(n=200)$

\begin{tabular}{|c|c|c|c|c|c|}
\hline \multirow[t]{2}{*}{ TTH Sub-types } & \multicolumn{3}{|c|}{ Thyroid Status } & \multirow[t]{2}{*}{ Total } & \multirow[t]{2}{*}{ P Value } \\
\hline & Subclinical Hypothyroidism & Overt Hypothyroidism & Normal Status & & \\
\hline Infrequent & 16 & 4 & 60 & 80 & \\
\hline Frequent & 10 & 4 & 46 & 60 & 0.0001 \\
\hline Chronic & 12 & 12 & 18 & 42 & \\
\hline Probable & 3 & 0 & 15 & 18 & \\
\hline Total & 41 & 20 & 139 & 200 & \\
\hline
\end{tabular}

*p value was measured by Pearson's Chi-square Test 
as having tension type headache (TTH). However, the underlying cause of TTH is uncertain in most of the cases. There are several studiesthat tried to find any link between hypothyroidism and $\mathrm{TTH}^{8-9}$. Two of them were undertaken on small sample population and found no link $^{11-12}$. Rest of them had found a variable number of hypothyroid cases among TTH patients. This study included 200 patients of different subtypes of TTH and tested for evidence of hypothyroidism.

All four sub-types of tension type headache were considered to include in the study, namely: Infrequent episodic or infrequent, frequent episodic or frequent, chronic sub-type and probable sub-type. Forty percent (40\%) patients in this study had infrequent TTH, 30\% had frequent episodic TTH, 21\% had chronic sub-type of TTH and 9\% patients had probable sub-type of TTH. In a study done by Khan et $\mathrm{al}^{8}$ in a tertiary care hospital of Kashmir, India they found among patients with TTH, 78 (47.6\%), 52(31.7\%) and 34(20.7\%) had infrequent episodic TTH, frequent episodic TTH and chronic TTH respectively ${ }^{8}$. Their study used the ICHD-2 criteria for TTH but did not include any patients with probable TTH. In this aspect this present study was a unique one as it had included probable sub-type of TTH along with other subtypes. However, when proportions of other sub-types of headache are compared with the study conducted by Khan et al 8 nearly equal results can be observed.

Prevalence of hypothyroidism in general population varies from country to country. Overall prevalence of subclinical hypothyroidism in general population is 4.0 to $10.0 \%$ cases $^{13}$. In India prevalence of hypothyroidism is $10.9 \%$ cases $^{14}$. In one study done in a community of Khulna district of Bangladesh prevalence of subclinical and overt hypothyroidism was found to be $6.59 \%$ and $4.97 \%$ respectively ${ }^{15}$. On the other hand, proportion of both subclinical (22\%) and overt hypothyroidism (7.2\%) was found high in TTH patients in the study done by Khan and colleagues 8 . In concordance with the later study, incidence of subclinical and overt hypothyroidism was found 20.5\% (41) and 10\% (20) respectively in this study. Jonathan R. Amy and Andrew J. Larner in their research did not find any patients of hypothyroidism in the sample population of headache? This was either because of small sample size (only 20 in the earlier study) or because small proportion of sample was tested for thyroid status only 13 among 119 patients in the later study. From above studies it can be inferred that incidence of hypothyroidism is more in TTH than normal people.

Among 80 infrequent TTH patients 20 had hypothyroidism and among 42 chronic TTH patients 24 had hypothyroidism. The difference is statistically highly significant $(p=0.0001)$. The difference in between frequent TTH and chronic TTH in relation to thyroid status (14 and 24 hypothyroid patients respectively) is also statistically significant ( $\mathrm{p}=0.001)$. These findings again go along with the findings of Khan et $a l^{8}$ who compared difference between chronic and infrequent sub-type and in between chronic and frequent sub-type. This implies that chronic TTH patients are statistically more likely to be found hypothyroid than both frequent episodic and infrequent episodic patients.

There were a number of limitations of the study which includes small sample size. Considering the limitation of the time, logistic supports, availability of patients and lack of sufficient fund, sample size was considered 200. Further case-control study is needed to confirm the consistency of the findings.

\section{Conclusion}

Headache particularly tension-type headache and hypothyroidism both affect the quality of life significantly. The study reveals positive correlation between two of them. However, without case-control study significant association between infrequent TTH and hypothyroidism cannot be established. However, from this study it is recommended that thyroid hormone level should be tested in each case of infrequent tension-type headache and hypothyroidism should be considered as a risk factor for primary headache disorder. More study is necessary for a more conclusive statement.

\section{References}

1. Anttila P. Tension-type headache in children and adolescents. Current pain and headache reports. 2004;8(6):500-4

2. Olesen J, Steiner TJ. The International classification of headache disorders, 2nd edn (ICDH-II). Journal of neurology, neurosurgery, and psychiatry, 2004;75:808-811

3. Olesen J. The international classification of headache disorders. Headache: The Journal of Head and Face Pain. 2008 May;48(5):691-3

4. Waldie K, Buckley J, Bull PN, Poulton R. Tension-type headache: A life-course review. Journal of Headache 2015;1(12):1-9

5. Jensen R. Pathophysiological mechanisms of tension-type headache: a review of epidemiological and experimental studies. Cephalalgia. 1999;19(6):602-21

6. Loder E, Rizzoli P. Tension-type headache. BMJ. 2008;336(7635):88-92

7. Jensen R, Stovner LJ. Epidemiology and comorbidity of headache. The Lancet Neurology. 2008;7(4):354-61

8. Khan HB, Shah PA, Bhat MH, Imran A. Association of hypothyroidism in patients with migraine and tension type 
headache disorders in Kashmir, North India. Neurology Asia. 2015;20(3):257-61

9. Larner AJ. Thyroid dysfunction and headache. Journal of Headache and Pain 2006;7(1):51-2

10. Bendtsen L. Drug and nondrug treatment in tension-type headache. Therapeutic advances in neurological disorders. 2009;2(3):155-61

11. Lima Carvalho MD, de Medeiros JS, Valença MM. Headache in recent onset hypothyroidism: Prevalence, characteristics and outcome after treatment with levothyroxine. Cephalalgia. 2017;37(10):938-46

12. Fumal A, Schoenen J. Tension-type headache: current research and clinical management. The Lancet Neurology. 2008;7(1):70-83
13. Gharib H, Tuttle RM, Baskin HJ, Fish LH, Singer PA, McDermott MT. Subclinical thyroid dysfunction: a joint statement on management from the American Association of Clinical Endocrinologists, the American Thyroid Association, and the Endocrine Society. Thyroid. 2005;15(1):24-8

14. Unnikrishnan AG, Kalra S, Sahay RK, Bantwal G, John M, Tewari N. Prevalence of hypothyroidism in adults: An epidemiological study in eight cities of India. Indian journal of endocrinology and metabolism. 2013;17(4):647

15. Paul AK, Miah SR, Mamun AA, Islam S. Thyroid disorders in Khulna district: a community based study. Bangladesh medical research council bulletin. 2006;32(3):66-71 